\title{
Spike Protein Undeformable Motif shared by SARS-CoV-2 and SARS-CoV: Flexible Conformations Predicted by using Deep Neural Network-based Programs of Supersecondary Structure Codes
}

\author{
Hiroshi Izumi ${ }^{1}$ \\ ${ }^{1}$ National Institute of Advanced Industrial Science and Technology
}

April 28, 2020

\begin{abstract}
A deep neural network-based program for sequence-based prediction of supersecondary structure codes (SSSCs), called SSSCPrediction (SSSCPred) was constructed. Furthermore, to predict the flexibility and conformational change of proteins, a comparison program of three deep-neural-network-based prediction systems (SSSCPred200, SSSCPred100, and SSSCPred) was developed. I compared the predicted and observed flexible conformations of SARS-CoV-2 and SARS-CoV spike proteins by using SSSCs and the comparison program. The SARS-CoV SSSC sequences of the receptor-binding motif predicted by the three deep-neural-network-based systems well reproduced those of the Protein Data Bank (PDB) data, including the structured loops. In contrast, the receptor-binding motif SSSCs of SARS-CoV-2 differs greatly from those of SARS-CoV, with that of SARS-CoV-2 being more flexible. Only one common identical motif (SSSC: SSSHSSHHHH) among all of the compared SSSC sequences, including predicted and observed ones, was found at the S2 subunit. This motif has an extremely rare and relatively undeformable conformation. The comparison program may be helpful to explore undeformable drug discovery targets of many unsolved protein structures.
\end{abstract}

\section{Hosted file}

SARS_CoV_2_Izumi_rev2.pdf available at https ://authorea.com/users/301438/articles/442751-spikeprotein-undeformable-motif-shared-by-sars-cov-2-and-sars-cov-flexible-conformations-predictedby-using-deep-neural-network-based-programs-of-supersecondary-structure-codes 